\title{
A TOPOLOGICAL EQUIVALENCE OF THE SINGULAR CARDINALS HYPOTHESIS
}

\author{
M. ISMAIL AND A. SZYMANSKI
}

(Communicated by Andreas R. Blass)

\begin{abstract}
We show, in particular, that the Singular Cardinals Hypothesis is equivalent to the following statement: for each metric space $X$ of cardinality greater than the continuum and of weigh of uncountable cofinality, the cardinality of $X$ equals the weight of $X$.
\end{abstract}

The Singular Cardinals Hypothesis, $\mathrm{SCH}$, states that if $\kappa$ is a singular cardinal and $2^{\mathrm{cf}(\kappa)}<\kappa$, then $\kappa^{\mathrm{cf}(\kappa)}=\kappa^{+}$(cf. [Je]). The aim of this note is to give a purely topological equivalence of $\mathrm{SCH}$.

Given a topological space $X$, let $w(X), d(X), s(X)$, and $t(X)$ denote, respectively, the weight of $X$, the density of $X$, the spread of $X$, and the tightness of $X$. For definitions of these and other concepts see [Ju].

Let $P$ denote the class of all regular $T_{1}$ topological spaces $X$ which satisfy the following two conditions:

(i) $s(X)=w(X)$;

(ii) for any closed subspace $Y$ of $X, d(Y)=w(Y)$.

All metrizable spaces are in $P$. More generally, every regular $T_{1}$ space with a point countable base satisfying condition (i) belongs to $P$. Furthermore, if $F=\operatorname{cl} F \subseteq X \in P$ and $s(F)=w(F)$, then $F \in P$ (we will use this fact without explicit mention in Cases I and II of our Theorem, below). Notice that

Lemma. If $X \in P$ and $A \subseteq X$, then $|\mathrm{cl} A| \leq|A|^{t(X)}$.

Proof. If $B \subseteq X$ and $|B| \leq t(X)$, then by (ii), $w(\mathrm{cl} B) \leq t(X)$. Hence $|\mathrm{cl} B| \leq$ $2^{t(X)}$. Since $\operatorname{cl} A=\bigcup\{\operatorname{cl} B: B \subseteq A$ and $|B| \leq t(X)\}$, it follows that

$$
|\mathrm{cl} A| \leq 2^{t(X)} \cdot|A|^{t(X)}=|A|^{t(X)} .
$$

Let (S) denote the following statement:

(S): If $X$ is a topological space such that $|X|>2^{t(X)}$ and $\operatorname{cf}(w(X))>t(X)$, then $|X|=w(X)$.

Received by the editors April 29, 1994 and, in revised form, July 11, 1994.

1991 Mathematics Subject Classification. Primary 54A25, 03E65, 03E10.

Key words and phrases. SCH, weight, tightness. 
Theorem. The following are equivalent:

(a) $\mathrm{SCH}$.

(b) If $X \in P$, then $X$ satisfies (S).

(c) If $X$ is a metrizable space, then $X$ satisfies (S).

Proof. (a) $\rightarrow$ (b). Let $X \in P$ be such that $|X|>2^{t(X)}$ and $\operatorname{cf}(w(X))>t(X)$. Let $\tau=t(X)$ and $\lambda=w(X)$. Let $D=\left\{x_{\alpha}: \alpha<\lambda\right\}$ be a dense subset of $X$. By the above lemma, $|X| \leq \lambda^{\tau}$. Since $|X|>2^{\tau}, \lambda>2^{\tau}$. For each $\beta<\lambda$, let $Z_{\beta}=\operatorname{cl}\left\{x_{\alpha}: \alpha<\beta\right\}$. If $x \in X$, then there exists a subset $B$ of $D$ such that $|B| \leq \tau$ and $x \in \operatorname{cl} B$. Since $\operatorname{cf}(\lambda)>\tau$, there exists $\beta<\lambda$ such that $B \subseteq \operatorname{cl}\left\{x_{\alpha}: \alpha<\beta\right\}$. Thus $x \in Z_{\beta}$ and therefore $\bigcup\left\{Z_{\alpha}: \alpha<\lambda\right\}=X$. To prove that $|X|=\lambda$ it is enough to show that $|X| \leq \lambda$; we do this by induction on $\lambda$.

If $\lambda=\left(2^{\tau}\right)^{+}$, then, by the preceding lemma, $\left|Z_{\beta}\right| \leq 2^{\tau}$, for each $\beta<\lambda$. Hence $|X| \leq 2^{\tau} \cdot \lambda=\lambda$.

Now let $\lambda>\left(2^{\tau}\right)^{+}$, and suppose (S) has been established for all spaces in $P$ of weight $<\lambda$.

Case I. $\lambda$ is a limit cardinal. Let $\left\{\lambda_{\alpha}: \alpha<\operatorname{cf}(\lambda)\right\}$ be an increasing sequence of regular cardinals converging to $\lambda$ such that $\lambda_{\alpha}>2^{\tau}$ for each $\alpha<\operatorname{cf}(\lambda)$. By (i), for each $\alpha<\operatorname{cf}(\lambda)$, there exists a discrete subset $Y_{\alpha}$ of $X$ such that $\left|Y_{\alpha}\right|=\lambda_{\alpha}$. Let $X_{\alpha}=\operatorname{cl}\left(Z_{\lambda_{\alpha}} \cup Y_{\alpha}\right)$, for each $\alpha<\operatorname{cf}(\lambda)$. Then $w\left(X_{\alpha}\right)=\lambda_{\alpha}$ and $X_{\alpha} \in P$. Also $\left|X_{\alpha}\right| \geq \lambda_{\alpha}>2^{\tau} \geq 2^{t\left(X_{\alpha}\right)}$ and $\operatorname{cf}\left(\lambda_{\alpha}\right)=\lambda_{\alpha}>\tau \geq t\left(X_{\alpha}\right)$. Therefore, by the inductive hypothesis, $\left|X_{\alpha}\right|=\lambda_{\alpha}$ for each $\alpha<\operatorname{cf}(\lambda)$. Since $X=\bigcup\left\{X_{\alpha}: \alpha<\operatorname{cf}(\lambda)\right\},|X| \leq \lambda$.

Case II. $\lambda=\kappa^{+}$, for some $\kappa$ such that $\operatorname{cf}(\kappa)>\tau$. By (i), there exists a discrete subset $Y$ of $X$ such that $|Y|=\kappa$. For each $\alpha, \kappa<\alpha<\lambda$, let $X_{\alpha}=\operatorname{cl}\left(Z_{\alpha} \cup Y\right)$. Then $w\left(X_{\alpha}\right)=\kappa$ and $X_{\alpha} \in P$. Since $\kappa^{+}=\lambda>\left(2^{\tau}\right)^{+}$, $\kappa>2^{\tau}$. Also, $\left|X_{\alpha}\right| \geq \kappa>2^{\tau} \geq 2^{t\left(X_{\alpha}\right)}$ and $\operatorname{cf}(\kappa)>\tau \geq t\left(X_{\alpha}\right)$. Therefore, by the inductive hypothesis, $\left|X_{\alpha}\right|=\kappa$ for each $\alpha$. Since $X=\bigcup\left\{X_{\alpha}: \kappa<\alpha<\lambda\right\}$, $|X| \leq \lambda$.

Case III. $\lambda=\kappa^{+}$, for some $\kappa$ such that $\operatorname{cf}(\kappa) \leq \tau$. Then $2^{\mathrm{cf}(\kappa)} \leq 2^{\tau}<\kappa$. Therefore, by $\mathrm{SCH}, \kappa^{\operatorname{cf}(\kappa)}=\kappa^{+}=\lambda$. Hence $\lambda^{\tau}=\kappa^{\operatorname{cf}(\kappa) \cdot \tau}=\kappa^{\tau}$. Again, by $\mathrm{SCH}$, it follows that $\kappa^{\tau}=\kappa^{+}$(cf. [Je, Lemma 8.1]). Thus $\lambda^{\tau}=\lambda$. Hence, by the preceding lemma, $|X| \leq \lambda$.

The implication $(b) \rightarrow(c)$ is obvious.

(c) $\rightarrow$ (a). In view of a theorem of Silver (cf. [Je, Theorem 23]), it is enough to show that SCH holds for singular cardinals of cofinality $\omega$. So let $\kappa$ be a singular cardinal such that $\operatorname{cf}(\kappa)=\omega$ and $2^{\omega}<\kappa$. Let $Y$ be a discrete space of cardinality $\kappa^{+}$, and let $X$ be the product of countably many copies of the space $Y, X=Y^{\omega}$. Then $X$ is metrizable, $w(X)=\kappa^{+}, t(X)=\omega$, and $|X|=\left(\kappa^{+}\right)^{\omega}>2^{\omega}$. Hence, by $(S),\left(\kappa^{+}\right)^{\omega}=\kappa^{+}$. Thus $\kappa^{\omega} \leq \kappa^{+}$. Also, by König's Theorem (cf. [Je, Theorem 17]), $\kappa^{\omega} \geq \kappa^{+}$. Hence $\kappa^{\omega}=\kappa^{+}$.

Example. Let $\left\{\kappa_{n}: n<\omega\right\}$ be any increasing sequence of cardinals $\geq 2^{\omega}$, for instance, $\kappa_{0}=2^{\omega}, \kappa_{n+1}=\kappa_{n}^{+}$for each $n<\omega$. We set $\lambda=\sup \left\{\kappa_{n}: n<\omega\right\}$. Consider $\kappa_{n}$ to be a discrete space, and let $X$ be the product of the discrete spaces $\kappa_{n}, X=\prod\left\{\kappa_{n}: n<\omega\right\}$. Then $X$ is a metrizable space (and thus $X \in P), w(X)=\lambda$, and $|X|=\lambda^{\omega}>\lambda$. Thus $w(X)<|X|$.

This example shows that the requirement in $(\mathrm{S})$ that $\operatorname{cf}(w(X))>t(X)$ is essential. 


\section{REFERENCES}

[Je] T. Jech, Set theory, Academic Press, New York, 1978.

[Ju] I. Juhász, Cardinal functions in topology—Ten years later, Math. Centre Tracts, Amsterdam, 1980.

Department of Mathematics, SlipPery Rock University, Slippery Rock, Pennsylvania 16057

E-mail address, M. Ismail: msi@sruvm. sru.edu

E-mail address, A. Szymanski: sruvm. sru.edu 